\title{
Pancreatitis aguda necrotizante con colecciones líquidas complicadas con fístulas cutáneas
}

\author{
J. MOLINA INFANTE, O. NÚÑEZ MARTÍNEZ, O. NOGALES RINCÓN, A. \\ PONFERRADA DÍAZ, G. M. BORREGO RODRÍGUEZ \\ Servicio de Aparato Digestivo. Hospital General Universitario Gregorio Marañón. \\ Madrid
}

\author{
ACUTE PANCREATITIS WITH FLUID COLLECTIONS COMPLICA- \\ TED WITH CUTANEOUS FISTULA
}

\section{RESUMEN}

La formación de colecciones líquidas peripancreáticas en el seno de una pancreatitis aguda es una complicación frecuente con buena evolución en la mayoría de los casos. Presentamos el caso de una mujer con pancreatitis aguda grave con colecciones líquidas a tensión que produjeron de manera espontánea fístulas cutáneas múltiples. No hemos encontrado datos en la literatura acerca de esta excepcional complicación. Revisamos el manejo terapéutico de las colecciones líquidas y fístulas pancreáticas

PALABRAS CLAVE: Pancreatitis aguda grave. Colecciones líquidas. Fístulas cutáneas.

\begin{abstract}
The presence of acute fluid collections is an habitual event in an acute pancreatitis, generally followed by a favourable outcome. We present a case about an ancient woman suffering from an acute necrotizing pancreatitis with tense fluid collections that produced espontaneously several cutaneous fistula. We find no report on literature about this uncommon complication. We revised clinical management of acute fluid collections and pancreatic fistulas.
\end{abstract}

KEY WORDS: Severe acute pancreatitis. Fluid collections. Cutaneous fistula.

Molina Infante J, Núñez Martínez O, Nogales Rincón O, Ponferrada Díaz A, Borrego Rodríguez GM. Pancreatitis aguda necrotizante con colecciones líquidas complicadas con fístulas cutáneas. An Med Interna (Madrid) 2003; 20: 413-415.

INTRODUCCIÓN

Las colecciones líquidas peripancreáticas en una pancreatitis aguda representan la reacción exudativa de la glándula ante el proceso inflamatorio local. En el contexto de una pancreatitis aguda moderada-grave aparecen en más del 50\% de los casos. No poseen pared propia y carecen de comunicación con el conducto pancreático principal en la mayoría de los casos, por lo que el líquido que contienen es un ultrafiltrado plasmático con valores mínimos de amilasa pancreática. Estas colecciones líquidas revierten de manera espontánea en alrededor del $65 \%$ de los casos y la persistencia de las colecciones más de 4 semanas aumenta la probabilidad de trasformación de las mismas en pseudoquistes pancreáticos $(1,2)$. Comunicamos el caso de una paciente con pancreatitis aguda que desarrolló de manera precoz y espontánea una excepcional complicación local.

\section{CASO APORTADO}

Mujer de 90 años con antecedentes personales de hipertensión arterial en tratamiento con furosemida, hipercolesterolemia sin tratamiento, accidente cerebrovascular isquémico con hemiparesia derecha residual, colectomía subtotal por obstrucción intestinal y anemia multifactorial con requerimientos trasfusionales periódicos.

Ingresó por dolor en hipogastrio de instauración brusca, irradiado en cinturón, de 48 horas de evolución, que se acompañaba de náuseas y vómitos biliosos. En la exploración física la paciente impresionaba de gravedad, tensión arterial $100 / 60 \mathrm{mmHg}$, temperatura de $37,9^{\circ} \mathrm{C}$, mal perfundida, sudorosa, taquicardia sinusal a $120 \mathrm{lpm}$ y taquipnea a $25 \mathrm{rpm}$. En la auscultación cardiopulmonar destacaba disminución del murmullo vesicular en base izquierda con semiología de derrame pleural. El abdomen estaba distendido, presentando edema con fóvea en flanco izquierdo y abolición completa de los ruidos hidroaéreos. Asimismo, era doloroso a la palpación profunda en hipogastrio palpándose en fosa ilíaca izquierda varias masas de con-

Trabajo aceptado: 30 de diciembre de 2002 
sistencia indurada, fluctuantes, con aumento de temperatura local, a tensión, sin signos de irritación peritoneal.

En las pruebas complementarias al ingreso destacaba una hemoglobina de $10 \mathrm{~g} / \mathrm{dl}$, leucocitos de $20.000 / \mathrm{mm}^{3}$ (95\% polimorfonucleares), plaquetas de $260.000 / \mathrm{mm}^{3}$, fibrinógeno $597 \mathrm{mg} / \mathrm{dl}$, glucosa 157 $\mathrm{mg} / \mathrm{dl}$, urea $145 \mathrm{mg} / \mathrm{dl}$, creatinina $2,45 \mathrm{mg} / \mathrm{dl}$, LDH $1433 \mathrm{UI} / \mathrm{l}$ y amilasa pancreática $6.864 \mathrm{UI} / \mathrm{l}$. Dada la gravedad del cuadro, se realizó TC abdominal urgente evidenciándose desestructuración morfológica de páncreas con afectación de la grasa peripáncreática y necrosis focal en cuerpo y cola pancreática, así como múltiples colecciones líquidas localizadas en el espacio pararrenal anterior izquierdo, epiplon mayor en el lado izquierdo y espacio subperitoneal que se extendían hacia la pared pélvica anterior contactando íntimamente con los planos musculares abdominales. La paciente presentó una evolución tórpida durante las 2 semanas posteriores, con fiebre de $38^{\circ} \mathrm{C}$ mantenida, íleo paralítico prolongado que precisó de soporte nutricional parenteral y aumento de número y tamaño de colecciones líquidas objetivable a la exploración física, especialmente en fosa ilíaca izquierda, donde la tensión parietal creó varios orificios fistulosos que drenaban líquido seroso grisáceo sin amilasa ni datos de infección en el análisis del mismo. Se realizó control radiológico con TC abdominal donde se observó aumento del número de colecciones pélvicas con múltiples trayectos fistulosos con drenaje externo en fosa iliaca izquierda (Fig. 1), por lo que se procedió a la colocación de drenaje percutáneo y tratamiento con octreótide a dosis de 100 microgramos cada 8 horas, comprobándose la disminución del tamaño de las colecciones a las 72 horas por TC abdominal.

Sin embargo, la evolución clínica de la paciente no fue favorable desarrollando shock séptico refractario a las medidas convencionales y fallo multiorgánico, motivo por el que falleció 5 días después de la colocación del drenaje percutáneo.

DISCUSIÓN

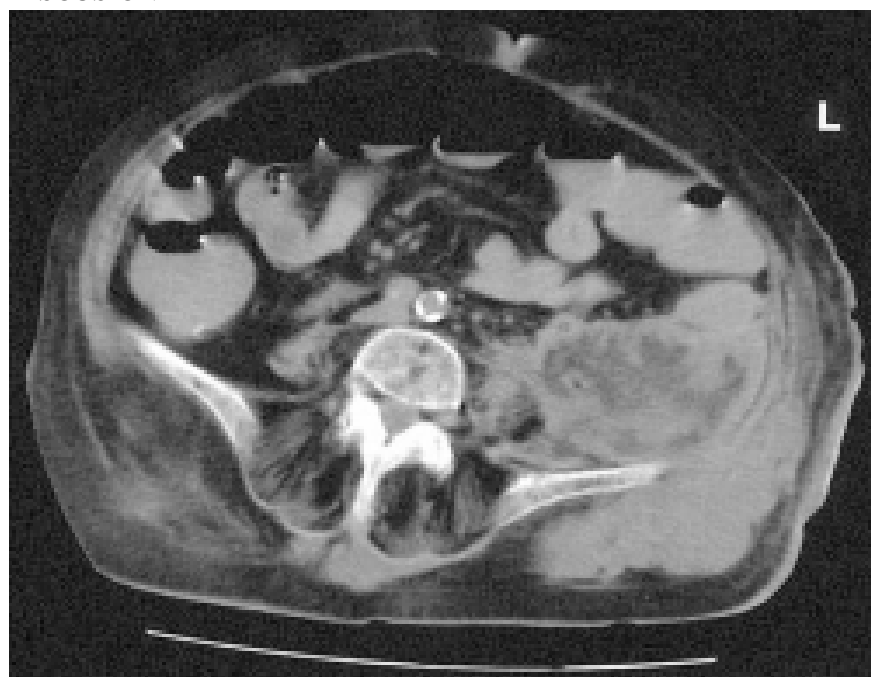

Fig. 1. TC abdominal donde podemos observar colección líquida en fosa ilíaca izquierda fistulizada a piel a través de múltiples trayectos fistulosos.

La pancreatitis aguda necrotizante es una entidad con una tasa global de mortalidad del $30 \%$, gravada por múltiples complicaciones sistémicas y locales $(1,2)$. Las muertes suelen ocurrir en dos fases bien definidas: una precoz (dos primeras semanas del cuadro clínico), debida a fallo multiorgánico en relación con el síndrome de respuesta inflamatoria sistémica (SRIS), y otra tardía, debida a complicaciones infecciosas locales y sistémicas $(2,3)$.

La existencia de colecciones líquidas en la fase aguda de la enfermedad no tiene implicaciones terapéuticas ni pronósticas dada la alta tasa de resolución espontánea (menor si existe disrupción del conducto pancreático); sin embargo, se debe realizar seguimiento a medio plazo (4-8 semanas) de estas colecciones por técnicas de imagen, habitualmente TC, ya que alrededor del $10-15 \%$ pueden encapsularse por tejido de granulación y formar un pseudoquiste (1). En pacientes con colecciones líquidas peripancreáticas sintomáticas (dolor abdominal, fiebre, leucocitosis, aumento del número de colecciones) se debe valorar la integridad del conducto de Wirsung mediante colangiopancreatografía retrógrada endoscópica (CPRE), que se encuentra interrumpida en un 30\% de las pancreatitis necrotizantes graves (4). La existencia de comunicación de las colecciones con el conducto pancreático no implica siempre manejo quirúrgico (5), por lo que se debe realizar una evaluación de la técnica terapéutica más adecuada (terapéutica endoscópica, drenaje percutáneo, cirugía...) en función de las características individuales de cada paciente. En nuestro caso, se optó por la alternativa menos agresiva dada su edad y patología de base, así como la tensión de las colecciones, aunque la colocación de endoprótesis por CPRE con drenaje transpapilar, en caso de haberse confirmado ruptura del Wirsung, podría haber sido una medida eficaz. De la misma manera, el uso precoz de octreótido subcutaneo en las pancreatitis agudas necrotizantes graves parece haber demostrado disminución de las complicaciones (fundamentalmente sepsis y distress respiratorio), de la mortalidad y de la estancia hospitalaria $(6,7)$, pero no hay evidencias sobre su papel en el tratamiento de las colecciones líquidas.

La aparición de fístulas cutáneas en enfermedades pancreáticas se relaciona con enfermedades pancreáticas primarias (pancreatitis aguda, pancreatitis crónica, abscesos, neoplasias, traumatismos) o secundarias a maniobras invasivas locales (cirugía páncreas o gastrointestinal, drenaje percutáneo, terapéutica endoscópica...) (8). El principal factor implicado en su génesis es la presencia de necrosis de parénquima pancreático con ruptura de los conductos pancreáticos y extravasación de enzimas pancreáticas y productos inflamatorios, lo que produce disección del tejido peripanceático con la consiguiente creación de trayectos fistulosos gastrointestinales o cutáneos (9). El tratamiento inicial consiste en nutrición parenteral y análogos de la somatostatina, debido a la marcada reducción que producen de la secreción pancreática e intestinal (7), valorando la asociación de tratamiento endoscópico o drenaje percutáneo en función de las características morfológicas del conducto de Wirsung y del tipo de enfermedad pancreática. Así, Kozarek (10) describió una serie de 17 pacientes con fístulas externas e internas a los que se les colocó una prótesis traspapilar por CPRE, con respuesta favorable en todos los casos excepto uno. El papel del tratamiento percutáneo está por determinar, aunque publicaciones recientes aportan tasas de éxito globales del $80 \%(9,11)$. Dada su elevada mortalidad $(22-29 \%)(12,13)$, la cirugía se debe reservar para casos seleccionados: hemorragia incontrolable, sepsis, peritonitis, fistulas colopancreáticas y casos refractarios a las medidas previas $(8,9)$.

En nuestro caso, nos encontramos con colecciones líquidas peripancreáticas no infectadas con fistulización externa espontánea. No hemos hallado datos en la literatura, mediante revisión Med-Line, respecto a este tipo de complicación, por lo que la actitud terapéutica adoptada se basó en las medidas previamente comentadas, aunque no disponíamos de eviden- 
cia científica de su eficacia.

Podemos concluir que la existencia de colecciones líquidas en una pancreatitis aguda es un evento clínico frecuente, que requiere revisión a medio plazo por técnicas de imagen para descartar la formación de pseudoquistes. Sin embargo, la

\section{Bibliografía}

1. Pitchhumoni CS, Agarwal N. Pancreatic pseudocysts. When and how should drainage be performed? Gastroenterol Clin N Am 1999; 28: 61539.

2. United Kingdom guidelines for the management of acute pancreatitis. Gut 1998; 42 (Supl. 2): S1-S13.

3. Baron TH, Morgan DE. Acute necrotizing pancreatitis. New Eng J Med 1999; 340: 1412-7.

4. Shash RJ, Martin SP. Endoscopic retrograde cholangiopancreatography in the diagnosis and management of pancreatic diseases. Current Gastroenterol Reports 2000; 2: 133-145.

5. Uomo G, Molino D, Visconti M, et al. The incidence of main pancreatic duct disruption in severe biliary pancreatitis. Am J Surg 1998; 176: 4952.

6. Paran H, Mayo A, Paran D, et al. Octreotide treatment in patients with severe acute pancreatitis. Dig Dis Sci 2000; 45: 2247-52.

7. Uhl W, Anghelacopoulos SE, Fiess H, et al. The role of octreotide and aparición espontánea de fístulas cutáneas debido a colecciones líquidas a tensión asociadas a una pancreatitis aguda grave es una complicación excepcional que no hemos hallado descrita en la literatura. somatostatin in acute and chronic pancreatitis. Digestion 1999; 60: $23-$ 31.

8. Ho HS, Frey CF. Gastrointestinal and pancreatic complications associated with severe pancreatitis. Arch Surg 1995; 130: 817-823.

9. Cabay JE, Boverie JH, Dondelinger RF. Percutaneous catheter drainage of external fistulas of the pancreatic ducts. Eur Radiol 1998; 8: 445-8.

10. Kozarek R. Endsocopic therapy of complete and partial pancreatic duct disruptions. Gastrointest Endosc Clin N Am 1998; 8: 39-53.

11. Cope C, Tuite C, Burke DR et al. Percutaneous management of chronic pancreatic duct strictures and external fistulas with long-term results. J Vasc Interv Radiol 2001; 12: 104-10.

12. Tsiotos G, Smith D, Sarr M. Incidence and management of pancreatic and enteric fistulas after surgical management of severe necrotizing pancreatitis. Arch Surg 1995; 130: 48-52.

13. Fielding GA, McLatchie GR, Wilson $\mathrm{C}$ et al. Acute pancreatitis and pancreatic fistula formation. Br J Surg 1989; 76: 1126-8. 